\title{
A Novel Single Layer Partially Reflection Surface for Broadband Fabry-Perot Resonator Antenna
}

\author{
Hanjing $\mathrm{Li}^{\mathrm{a}}$, Yuwen Wang ${ }^{\mathrm{b}}$, Qiang Shuc ${ }^{\mathrm{c}}$, and Zekun $\mathrm{Li}^{\mathrm{d}}$ \\ School of Astronautics and Aeronautics, University of Electronic Science and Technology of China, \\ Chengdu, China

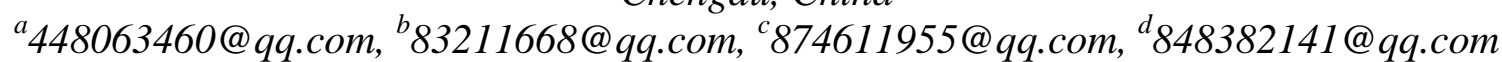

Keywords: Single Layer; Fabry-Perot resonator antenna; PRS.

\begin{abstract}
In this paper, a novel partially reflction surface (PRS) is proposed. It is a single layer periodic array, which is composed of two folded long dipoles, two rectangular patches and four short dipoles. Two folded long dipoles are main resonant cells, which provides positive reflection coefficient phase gradient. In addition, two rectangular patches and four short dipoles can improve reflection coefficient magnitude to some extent. Compared with other papers, the novelty of this paper is that the proposed structure is single array, but the most proposed structure of other papers are dual layer periodic patch arrays. The reflection coefficient phase of this PRS increases linearly with operating frequency from $6.1 \mathrm{GHz}$ to $6.7 \mathrm{GHz}$. The simulated results show that the reflection coefficient phase of this PRS is completely consistent with the theoretical reflection coefficient phase, the reflection coefficient magnitude of this PRS ias about 0.728 at $6.48 \mathrm{GHz}$. Therefore, it can be used as superstrate of the Fabry-Perot resonator antenna for enhancing its half-power fractional bandwidth and gain.
\end{abstract}

\section{Introduction}

With the development of wireless communication technology, the demands for highly directive antennas are becoming more and more stringent ${ }^{[1]}$. Traditional technologies for obtaining high gain antennas are mainly based on reflectors ${ }^{[2]}$, dielectric lenses ${ }^{[3]}$, waveguide horns ${ }^{[4]}$ and microstrip antenna arrays ${ }^{[5]}$, which may suffer from design complexity, high cost and power losses in the feeding network ${ }^{[6]}$. In recent decades, electromagnetic band gap (EBG) structures have attracted a lot of interest from the electromagnetic community for applications in controlling electromagnetic waves ${ }^{[7]}$. Among them, one particular application concerns the realization of high gain Fabry-Perot (FP) resonator antennas, also known as EBG resonator antennas ${ }^{[8]}$. A conventional FP resonator antenna is formed by placing an EBG structure as a partially reflective surface (PRS) at a proper distance from a ground plane, which creates an air-filled cavity between the PRS and the ground plane, and fed by a small antenna or an array. The main characteristics of the antenna, such as its operating frequency, directivity, gain bandwidth and radiation patterns, are determined by the property of the PRS. The operating frequency depends on the following equation ${ }^{[7]}$, 


$$
f=\frac{c}{4 \pi h}\left(\varphi_{p}+\varphi_{g}-2 N \pi\right), \quad N=0,1,2, \cdots
$$

Where $c$ is the velocity of light, $\varphi_{p}$ and $\varphi_{g}$ are the reflection coefficient phases of the PRS and the ground, respectively. The $h$ is the distance between the PRS and the ground plane. narrow bandwidth due to it's typical narrowband resonant cavity. To overcome this, three methods to enhance the gain bandwidth have been proposed so far. The first one employed an antenna array instead of a single antenna as the excitation to feed an FP resonator antenna ${ }^{[9]}$, where an enhanced $3 \mathrm{~dB}$ gain bandwidth can be obtained. However, a complex feeding network is indispensable for this approach, losing the advantages of simplicity and high radiation efficiency of an FP resonator antenna over a conventional array antenna. In the second method ${ }^{[10]}$, a mushroom-type high impedance surface (HIS) with size-tapered patches and a single layer printed FSS were applied as the ground plane and the PRS, respectively, in the design of a wideband FP resonator antenna. With the help of the elaborated HIS, an enhanced $3 \mathrm{~dB}$ gain bandwidth also can be obtained. The third method was based on the analysis in [11]. In [11], for a FP resonator antenna, when a PEC ground plane is applied ( $\left.\varphi_{g}=\pi\right)$, (1) can be rearranged as:

$$
\varphi_{p}=\frac{4 \pi h}{c} f+(2 N-1) \pi, \quad N=0,1,2, \cdots
$$

The formula (2) indicates that a wideband FP resonator antenna could be obtained by using a PRS with a positive reflection phase gradient over operating frequency. In fact, the PRSs with a positive reflection coefficient phase gradient have been designed in [12] and 13]. Both of which are dual-layer periodic patch arrays, and have been used successfully as superatrate to improve the bandwidth of FP resonator antennas.

In this paper, a novel PRS is proposed, which is a single layer periodic patch array. This also shows positive reflection coefficient phase gradient. In operating frequency band, its reflection coefficient phase is completely consistent with theoretical reflection coefficient phase, and it shows this PRS can be used as the superstrate of FP resonator antenna to improve its bandwidth. Next, this structure will be fabricated and experimentally verified.

\section{Proposed Partially Reflection Surface Geometry}

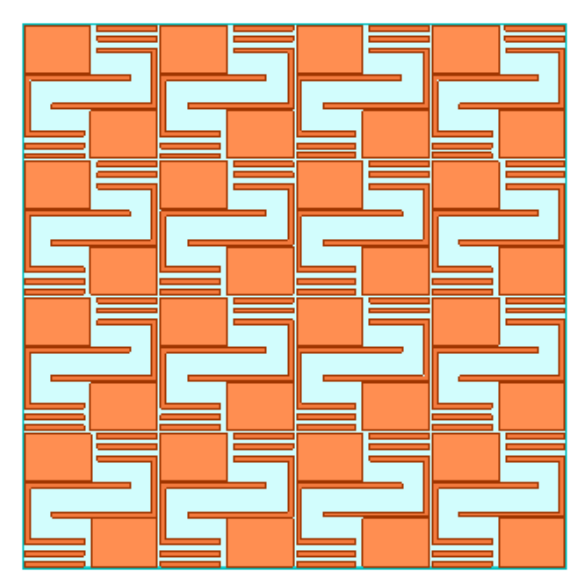

(a)

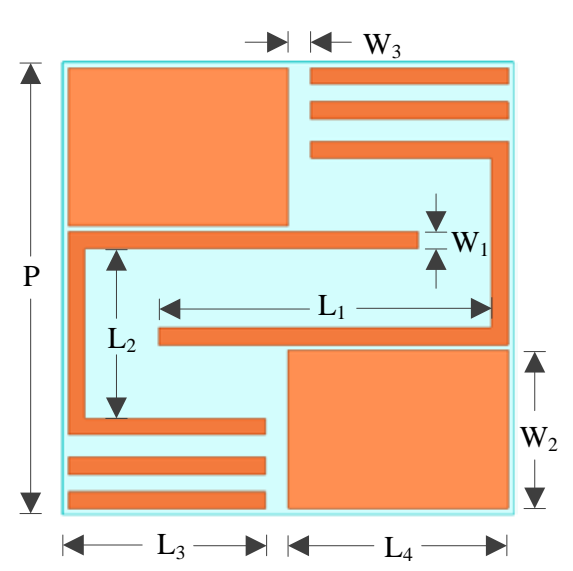

(b)

Figure 1. Geometry of single layer PRS. (a) A complete view of the proposed PRS, (b) Unit of PRS.

Geometry of the single layer PRS is shown in Fig. 1. Its unit cell consists of two folded long 
strips, two rectangular patches and four short strips. Two folded long strips are main resonant cells, which provide positive reflection coefficient phase gradient. Additionally, two rectangular patches and four short strips can improve reflection coefficient magnitude to some extent, but, these also decrease the bandwidth of PRS. According to the design requirements, the dimensions of those sections can be adjusted. Rogers RT/duroid 6010 slab with thickness of $1.6 \mathrm{~mm}$ was used as a substrate material.

Lease follow these instructions as carefully as possible so all articles within a conference have the same style to the title page. This paragraph follows a section title so it should not be indented.

The PRS design parameters, as defined in Figure 1, are:

$$
\begin{gathered}
P=8.0 \mathrm{~mm}_{1}=6.2 \mathrm{~mm}, \mathrm{~L}_{2}=2.6 \mathrm{~mm}, \mathrm{~L}_{3}=3.0 \mathrm{~mm}, \\
\mathrm{~L}_{4}=3.9 \mathrm{~mm}, W_{1}=0.2 \mathrm{~mm}, W_{2}=3.1 \mathrm{~mm} .
\end{gathered}
$$

\section{Simulation Results}

The presented PRS (Figure 1) has been modeled in Ansys HFSS software, and simulated reflection coefficient magnitude and phase are plotted in Figure 2 and Figure 3, respectively. From Figure 2 , it can be seen that, at $6.48 \mathrm{GHz}$, the reflection coefficient magnitude of this PRS is about 0.728. From Figure 3, it can be observed that, this PRS has positive reflection coefficient phase gradient from $6.1 \mathrm{GHz}$ to $6.7 \mathrm{GHz}$.

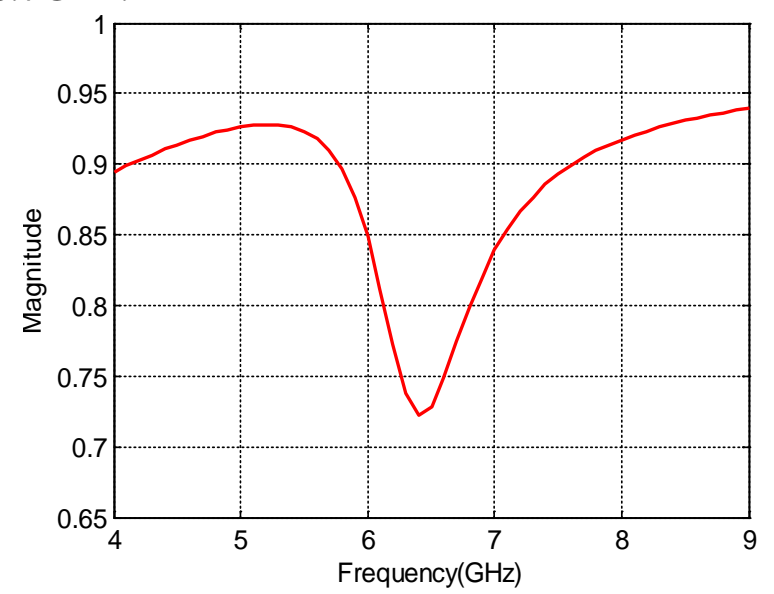

Figure 2. Reflection coefficient magnitude of the proposed PRS.

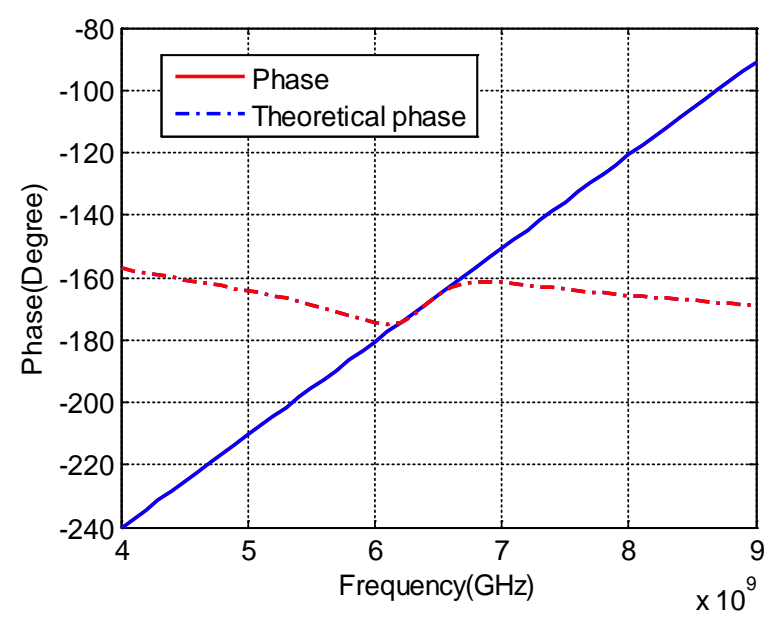

Figure 3. Reflection coefficient phase of the proposed PRS. 
The reflection coefficient phase of this PRS is completely consistent with theoretical reflection coefficient phase in operating frequency band $(6.1 \mathrm{GHz}$ to $6.7 \mathrm{GHz})$. It can be estimated that a FP resonator antenna with this PRS (Figure 1) will show high gain performance from 6.1-6.7GHz, however it is matter of current investigation.

\section{Conclusions and Future Study}

A novel single layer partially reflecting surface (PRS) is presented. The simulated results show that in the operating frequency band, the reflection coefficient phase of this partially reflection surface is completely consistent with the theoretical reflection coefficient phase, therefore it can be used as the superstrate of Fabry-Perot resonator antenna. This can help to achieve high gain and wideband in operating frequency band, simultaneously.

\section{References}

[1] S. W. Qu and K. B. Ng, “Wideband millimeter-wave cavity-backed bowtie antenna,” Progr. Electromagn. Res., vol. 133, pp. 477-493, 2013.

[2] P. Fei, Y. Qi, and Y.-C. Jiao, "Wide slot loop antenna with distance-adjustable back-reflector for multiple narrowband antennas replacement,” Progr. Electromagn. Res., vol. 135, pp. 563-581, 2013.

[3] C. Z. Hua, X. D.Wu, N. Yang, and W.Wu, "Millimeter-wave homogenous

[4] O. Xu, "Diagonal horn Gaussian efficiency enhancement by dielectric loading for submillimeter wave application at $150 \mathrm{GHz}$," Progr. Electromagn.

[5] R. Camblor-Diaz, S. Ver-Hoeye, C. Vazquez-Antuna, G. R. Hotopan, M. G. Fernandez, and F. Las-Heras, "Submillimeter wave frequency scanning 81 antenna array,” Progr. Electromagn. Res., vol. 132, pp. 215-232, 2012.

[6] Y. Lee, X. Lu, Y. Hao, S. Yang, J. Evans, and C. G. Parini, "Low-profile directive millimeter-wave antennas using free-formed three-dimensional (3-D) electromagnetic bandgap structures,” IEEE Trans. Antennas Propag., vol. 57, no. 10, pp. 2893-2903, Oct. 2009.

[7] L. Moustafa and B. Jecko, “EBG structure with wide defect band for broadband cavity antenna applications,” IEEE Antennas Wireless Propag. Lett., vol. 7, pp. 693-696, 2008.

[8] L. Leger, C. Serier, R. Chantalat, M. Thevenot, T. Monediere, and B. Jecko, "1-D dielectric EBG resonator antenna design,” Annal. Télécommun.,

[9] A.Weily, K. P. Esselle, T. S. Bird, and B. C. Sanders, "Dual resonator 1-D EBG antenna with slot array feed for improved radiation bandwidth,” IET Microwaves, Antennas Propag., vol. 1, no. 1, pp. 198-203, Feb. 2007.

[10] Z.-H. Wu and W.-X. Zhang, “Broadband printed compound air-fed array antennas," IEEE Antennas Wireless Propag. Lett., vol. 9, pp. 187-190, 2010.

[11] A. P. Feresidis and J. C. Vardaxoglou, "High gain planar antenna using optimized partially reflective surfaces," IEE Proc. Microwaves, Antennas Propag., vol. 148, no. 6, pp. 345-350, Dec. 2001.

[12] N. Z. Wang, Q. Liu, C.Y. Wu and L. Talbi, "Wideband Fabry-Perot Resonator Antenna with Two Complementary FSS Layers,” IEEE Trans. On Antennas and Propag., Vol. 62, no. 5, pp. 2463-2470, May 2014.

[13] Y. H. Ge, K. P. Esselle and T.S. Bird, "The Use of Simple Thin Partially Reflective Surfaces with Positive Reflection Phase Gradients to Design Wideband, Low-Profile EBG Resonator Antennas," IEEE Trans. On Antennas and Propag., Vol. 60, no. 2, pp.743-749, Feb. 2012. 